\title{
On Some Small Classical Ramsey Numbers
}

\author{
Geoffrey Exoo \\ Department of Mathematics and Computer Science \\ Indiana State University \\ Terre Haute, IN 47809 \\ ge@cs.indstate.edu
}

Submitted: Feb 17, 2013; Accepted: Mar 13, 2013; Published: Mar 24, 2013

Mathematics Subject Classifications: 05C55,05D10

\begin{abstract}
This note is a report on a computer investigation of some small classical Ramsey numbers. We establish new lower bounds for the classical Ramsey numbers $R(3,11)$ and $R(4,8)$. In the first case, the bound is improved from 46 (a record that had stood for 46 years) to 47 ; and in the second case the bound is improved from 57 to 58.
\end{abstract}

The classical Ramsey number $R(s, t)$ is the smallest integer $n$ such that in any twocoloring of the edges of $K_{n}$ there is a monochromatic copy of $K_{s}$ in the first color or a monochromatic copy of $K_{t}$ in the second color. A comprehensive summary of the current state of the art can be found in the dynamic survey on Small Ramsey Numbers [10].

In this note we present constructions that improve the lower bounds for the Ramsey numbers $R(3,11)$ and $R(4,8)$, and then describe the the modification in our search algorithm that led to the improvement in the $R(3,11)$ bound. Listings for these colorings are given at the end of this paper, and can also be found at the authors web site [4].

\section{$1 \mathrm{R}(3,11)$}

The best published bounds for $R(3,11)$ are $46 \leqslant R(3,11) \leqslant 50$. The lower bound of 46 was established 46 years ago [8], whereas the upper bound is very recent [7]. The graph that established the lower bound is the circle graph $45(1,3,5,12,19)$. In this note we present a graph which improves the lower bound by one.

Theorem 1. $R(3,11) \geqslant 47$. 
Proof. The proof is given by the coloring of $K_{46}$ which can be derived from Listing 1 at the end of the paper, wherein the adjacency list for the color one graph in a $(3,11)$-coloring of $K_{46}$ is given.

In all, we found 143447 pairwise non-isomorphic $(3,11)$-colorings of $K_{46}$. Of these, 141829 have a trivial automorphism group, and 1618 have exactly one non-trivial automorphism. The number of edges in these graphs that are assigned color one ranges from 217 to 228. Note that a 10-regular graph of order 46 has 230 edges. Adjacency matrices for several of these colorings can be found at the authors web site [4].

\section{$2 \mathrm{R}(3,10)$}

An effort was also made to improve the lower bound for $R(3,10)$. The current bounds in this case are $40 \leqslant R(3,10) \leqslant 42$. The lower bound was established in [1] and the upper bound was recently established in [7].

We were able to come tantilizingly close to a new lower bound, finding 810 pairwise non-isomorphic colorings of $K_{40}$ with exactly one monochromatic triangle in color one and no monochromatic copies of $K_{10}$ in color two.

In all we generated thousands of $(3,10)$-colorings of $K_{39}$. Using these colorings as a starting point, Jan Goedgebeur [6] has created a catalog of nearly $50000000(3,10)$ colorings of $K_{39}$.

\section{$3 \mathrm{R}(4,8)$}

In [5], Fujita used the SAT solver miniSat to improve the lower bound on $R(4,8)$ from 56 to 57 . Using Fujita's graph as a starting point, we were able to improve the bound to 58 using the method outlined in [3].

Theorem 2. $R(4,8) \geqslant 58$.

Proof. The proof is given by the coloring of $K_{57}$ which can be derived from listing 2, at the end of this note. This listing shows the adjacency list for the color one graph in a two coloring of $K_{57}$.

\section{The Construction Algorithm}

The method used to find our $R(3,11)$ colorings is similar to the method described in [3], with an important difference, which we now describe.

In general, the goal of the algorithm is to produce a $(s, t)$-coloring of $K_{n}$. One begins with a coloring obtained either randomly ${ }^{1}$ or from a known good coloring of a smaller

\footnotetext{
${ }^{1}$ To be more precise, we might replace random by pseudo-random.
} 
complete graph. Then each edge is examined, in random order, and counts are made of the number of $K_{s}$ subgraphs in color one that would result if the edge were assigned color one, and of the number of $K_{t}$ subgraphs that would result if the edge were assigned color two.

When dealing with diagonal Ramsey numbers (where $s=t$ ) one can simply compare the counts, choosing the better color most of the time, but choosing the other with a small probability (usually depending on the difference in the counts). This would be a typical implementation of simulated annealing [9].

For off-diagonal Ramsey numbers, there is additional issue. One needs to determine how much weight to assign to a $K_{s}$ in color 1 as a opposed to a $K_{t}$ in color 2. Empirical evidence suggests that the weights should be approximately inversely proportional to the $3 / 2$-power of the number of vertices in the complete subgraphs that one is trying to avoid. So in the case of $R(3,11)$, each monochromatic $K_{3}$ in color one would count $(11 / 3)^{1.5} \approx 7$ times as much as a monochromatic $K_{11}$ in color two. This is roughly the way subgraphs weights were assigned in [1], [2], and [3] to obtain new Ramsey bounds.

As with all randomized search methods, the essential problem here is how to avoid getting stuck in local minima. Typically this is done by occasionally picking the edge that produces the largest (weighted) subgraph count, instead of the smallest. In the algorithm that produced the $(3,11)$-coloring presented here, we added a second method. The relative weights of the two types of monochromatic subgraphs were changed at regular intervals. In this particular case, the weight of a monochromatic $K_{11}$ in color two was fixed at 1 , while the weight of a monochromatic $K_{3}$ in color one was allowed to vary from 1 to 13 . Within this range the $K_{3}$ weight was determined by a piecewise linear sawtooth function of time, except that when a new best coloring was achieved (measured by an unweighted subgraph count), the $K_{3}$ weight returned to the minimum value (1).

\section{Adjacency Lists}

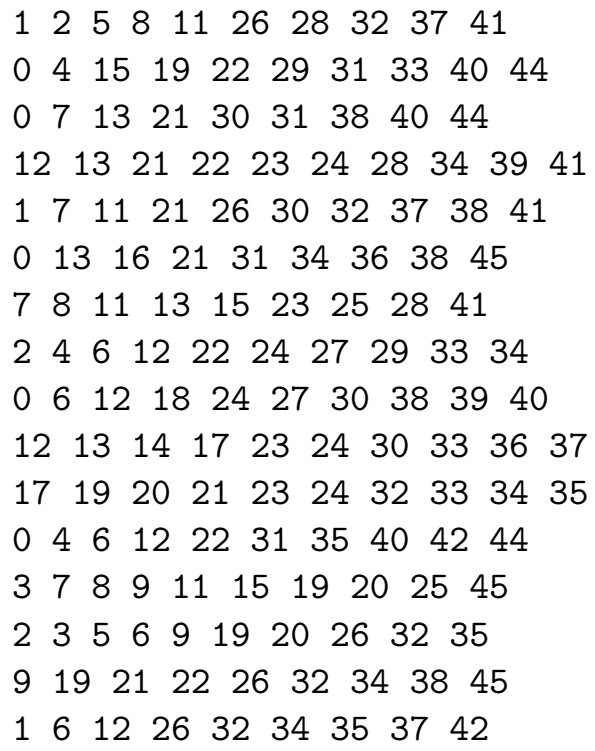

$\begin{array}{llllllllll}1 & 2 & 5 & 8 & 11 & 26 & 28 & 32 & 37 & 41\end{array}$

$\begin{array}{llllllllll}0 & 4 & 15 & 19 & 22 & 29 & 31 & 33 & 40 & 44\end{array}$

$\begin{array}{lllllllll}0 & 7 & 13 & 21 & 30 & 31 & 38 & 40 & 44\end{array}$

$\begin{array}{llllllllll}12 & 13 & 21 & 22 & 23 & 24 & 28 & 34 & 39 & 41\end{array}$

$\begin{array}{llllllllll}1 & 7 & 11 & 21 & 26 & 30 & 32 & 37 & 38 & 41\end{array}$

$\begin{array}{lllllllll}0 & 13 & 16 & 21 & 31 & 34 & 36 & 38 & 45\end{array}$

$\begin{array}{lllllllll}7 & 8 & 11 & 13 & 15 & 23 & 25 & 28 & 41\end{array}$

$\begin{array}{llllllllll}2 & 4 & 6 & 12 & 22 & 24 & 27 & 29 & 33 & 34\end{array}$

$\begin{array}{llllllllll}0 & 6 & 12 & 18 & 24 & 27 & 30 & 38 & 39 & 40\end{array}$

$\begin{array}{llllllllll}12 & 13 & 14 & 17 & 23 & 24 & 30 & 33 & 36 & 37\end{array}$

$\begin{array}{lllllllllll}17 & 19 & 20 & 21 & 23 & 24 & 32 & 33 & 34 & 35\end{array}$

$\begin{array}{llllllllll}0 & 4 & 6 & 12 & 22 & 31 & 35 & 40 & 42 & 44\end{array}$

$\begin{array}{llllllllll}3 & 7 & 8 & 9 & 11 & 15 & 19 & 20 & 25 & 45\end{array}$

$\begin{array}{llllllllll}2 & 3 & 5 & 6 & 9 & 19 & 20 & 26 & 32 & 35\end{array}$

$\begin{array}{lllllllll}9 & 19 & 21 & 22 & 26 & 32 & 34 & 38 & 45\end{array}$

$\begin{array}{lllllllll}1 & 6 & 12 & 26 & 32 & 34 & 35 & 37 & 42\end{array}$ 


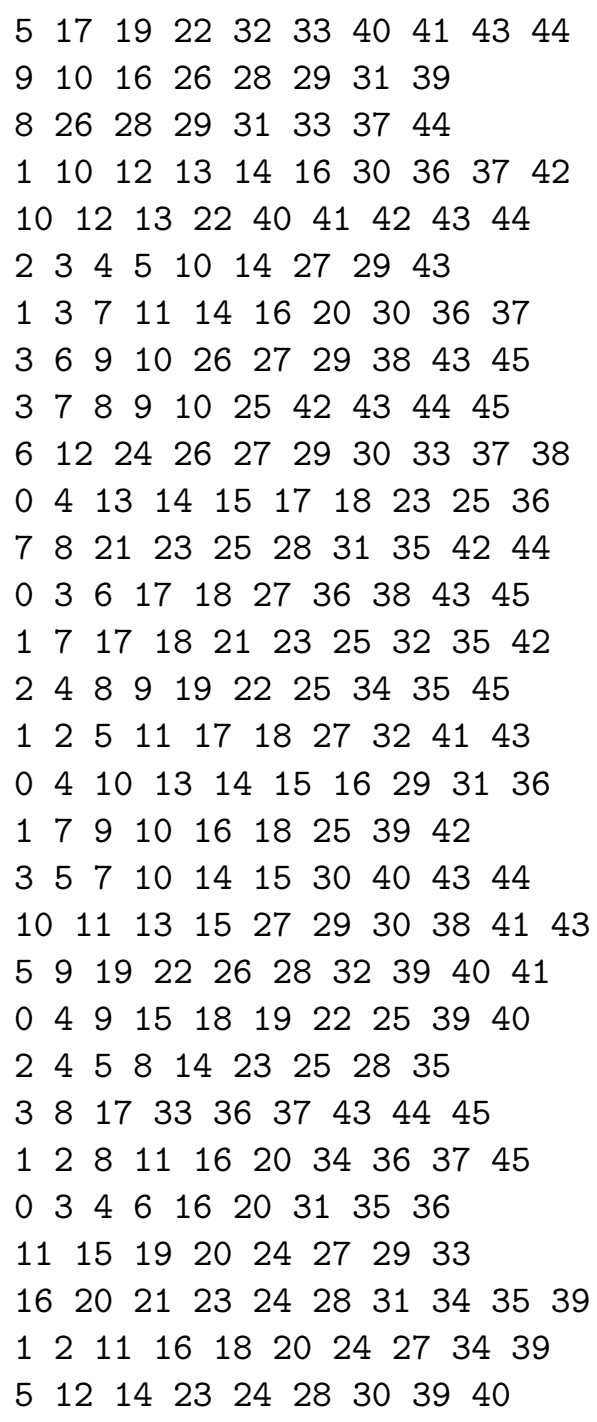

Listing 1. The Color 1 Graph in a $(3,11)$-coloring of $K_{46}$.

$\begin{array}{llllllllllllllllllll}2 & 3 & 5 & 6 & 10 & 11 & 12 & 19 & 22 & 23 & 25 & 26 & 36 & 37 & 42 & 43 & 45 & 46 & 50 & 56\end{array}$

$\begin{array}{lllllllllllllllllllll}3 & 4 & 6 & 7 & 11 & 12 & 13 & 19 & 23 & 24 & 25 & 26 & 27 & 37 & 38 & 43 & 44 & 46 & 47 & 50 & 53\end{array}$

$\begin{array}{lllllllllllllllllll}0 & 4 & 5 & 7 & 8 & 12 & 13 & 14 & 24 & 25 & 27 & 28 & 38 & 39 & 44 & 45 & 47 & 53 & 56\end{array}$

$\begin{array}{lllllllllllllllllll}0 & 1 & 5 & 6 & 8 & 9 & 14 & 15 & 25 & 26 & 28 & 29 & 39 & 40 & 41 & 45 & 46 & 48 & 55\end{array}$

$\begin{array}{llllllllllllllllllll}1 & 2 & 6 & 7 & 9 & 10 & 14 & 15 & 16 & 26 & 27 & 29 & 30 & 40 & 41 & 42 & 46 & 47 & 51 & 54\end{array}$

$\begin{array}{lllllllllllllllllll}0 & 2 & 3 & 7 & 8 & 10 & 11 & 16 & 17 & 27 & 28 & 30 & 31 & 41 & 42 & 43 & 47 & 54 & 55\end{array}$

$\begin{array}{lllllllllllllllllll}0 & 1 & 3 & 4 & 8 & 9 & 11 & 12 & 17 & 18 & 28 & 29 & 31 & 32 & 42 & 43 & 44 & 53 & 55\end{array}$

$\begin{array}{lllllllllllllllllll}1 & 2 & 4 & 5 & 9 & 10 & 12 & 13 & 18 & 29 & 30 & 32 & 33 & 43 & 44 & 45 & 52 & 53 & 54\end{array}$

$\begin{array}{llllllllllllllllllll}2 & 3 & 5 & 6 & 10 & 11 & 13 & 14 & 20 & 30 & 31 & 33 & 34 & 44 & 45 & 46 & 50 & 52 & 54 & 56\end{array}$

$\begin{array}{llllllllllllllllllll}3 & 4 & 6 & 7 & 11 & 12 & 13 & 14 & 15 & 20 & 21 & 31 & 32 & 34 & 35 & 45 & 46 & 47 & 50 & 53\end{array}$

$\begin{array}{lllllllllllllllllll}0 & 4 & 5 & 7 & 8 & 12 & 13 & 15 & 16 & 21 & 22 & 32 & 33 & 35 & 36 & 46 & 47 & 49 & 53\end{array}$

$\begin{array}{llllllllllllllllllll}0 & 1 & 5 & 6 & 8 & 9 & 13 & 14 & 16 & 17 & 22 & 23 & 33 & 34 & 36 & 37 & 47 & 49 & 50 & 55\end{array}$

$\begin{array}{llllllllllllllllllllll}0 & 1 & 2 & 6 & 7 & 9 & 10 & 14 & 15 & 16 & 17 & 18 & 23 & 24 & 34 & 35 & 37 & 38 & 48 & 50 & 51 & 55\end{array}$ 


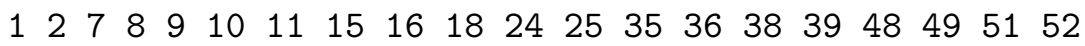

$\begin{array}{lllllllllllllllllll}2 & 3 & 4 & 8 & 9 & 11 & 12 & 16 & 17 & 19 & 20 & 25 & 26 & 36 & 37 & 39 & 40 & 49 & 52\end{array}$

$\begin{array}{llllllllllllllllllll}3 & 4 & 9 & 10 & 12 & 13 & 17 & 18 & 19 & 20 & 21 & 26 & 27 & 37 & 38 & 40 & 41 & 51 & 52 & 53\end{array}$

$\begin{array}{lllllllllllllllllll}4 & 5 & 10 & 11 & 12 & 13 & 14 & 18 & 21 & 22 & 27 & 28 & 38 & 39 & 41 & 42 & 52 & 53 & 56\end{array}$

$\begin{array}{lllllllllllllllllll}5 & 6 & 11 & 12 & 14 & 15 & 19 & 20 & 22 & 23 & 27 & 28 & 29 & 39 & 40 & 42 & 43 & 48 & 52\end{array}$

$\begin{array}{lllllllllllllllllll}6 & 7 & 12 & 13 & 15 & 16 & 19 & 20 & 21 & 23 & 24 & 28 & 29 & 30 & 40 & 41 & 43 & 44 & 48\end{array}$

$\begin{array}{llllllllllllllllll}0 & 1 & 14 & 15 & 17 & 18 & 28 & 29 & 31 & 32 & 37 & 45 & 48 & 49 & 51 & 53 & 54 & 56\end{array}$

$\begin{array}{lllllllllllllllllll}8 & 9 & 14 & 15 & 17 & 18 & 22 & 23 & 25 & 26 & 30 & 31 & 32 & 42 & 43 & 45 & 46 & 48 & 50\end{array}$

$\begin{array}{lllllllllllllllllll}9 & 10 & 15 & 16 & 18 & 23 & 24 & 26 & 27 & 31 & 32 & 33 & 43 & 44 & 46 & 47 & 50 & 52 & 56\end{array}$

$\begin{array}{lllllllllllllllllll}0 & 10 & 11 & 16 & 17 & 20 & 24 & 25 & 27 & 28 & 32 & 33 & 34 & 44 & 45 & 47 & 52 & 55 & 56\end{array}$

$\begin{array}{lllllllllllllllllll}0 & 1 & 11 & 12 & 17 & 18 & 20 & 21 & 25 & 26 & 28 & 29 & 33 & 34 & 35 & 45 & 46 & 53 & 55\end{array}$

$\begin{array}{llllllllllllllllllll}1 & 2 & 12 & 13 & 18 & 21 & 22 & 26 & 27 & 29 & 30 & 34 & 35 & 36 & 46 & 47 & 49 & 52 & 53 & 54\end{array}$

$\begin{array}{lllllllllllllllllllll}0 & 1 & 2 & 3 & 13 & 14 & 20 & 22 & 23 & 27 & 28 & 30 & 31 & 35 & 36 & 37 & 47 & 49 & 52 & 54 & 55\end{array}$

$\begin{array}{lllllllllllllllllll}0 & 1 & 3 & 4 & 14 & 15 & 20 & 21 & 23 & 24 & 28 & 29 & 31 & 32 & 37 & 38 & 49 & 50 & 55\end{array}$

$\begin{array}{lllllllllllllllllll}1 & 2 & 4 & 5 & 15 & 16 & 17 & 21 & 22 & 24 & 25 & 29 & 30 & 32 & 33 & 38 & 39 & 50 & 54\end{array}$

$\begin{array}{llllllllllllllllllll}2 & 3 & 5 & 6 & 16 & 17 & 18 & 19 & 22 & 23 & 25 & 26 & 30 & 31 & 33 & 34 & 39 & 40 & 50 & 54\end{array}$

$\begin{array}{lllllllllllllllllll}3 & 4 & 6 & 7 & 17 & 18 & 19 & 23 & 24 & 26 & 27 & 31 & 32 & 34 & 35 & 40 & 41 & 51 & 52\end{array}$

$\begin{array}{lllllllllllllllllll}4 & 5 & 7 & 8 & 18 & 20 & 24 & 25 & 27 & 28 & 32 & 33 & 35 & 36 & 41 & 42 & 49 & 51 & 52\end{array}$

$\begin{array}{lllllllllllllllllll}5 & 6 & 8 & 9 & 19 & 20 & 21 & 25 & 26 & 28 & 29 & 33 & 34 & 36 & 37 & 42 & 43 & 49 & 52\end{array}$

$\begin{array}{llllllllllllllllllll}6 & 7 & 9 & 10 & 19 & 20 & 21 & 22 & 26 & 27 & 29 & 30 & 34 & 35 & 37 & 38 & 43 & 44 & 49 & 56\end{array}$

$\begin{array}{lllllllllllllllllll}7 & 8 & 10 & 11 & 21 & 22 & 23 & 27 & 28 & 30 & 31 & 35 & 36 & 38 & 39 & 44 & 45 & 48 & 49\end{array}$

$\begin{array}{lllllllllllllllllll}8 & 9 & 11 & 12 & 22 & 23 & 24 & 28 & 29 & 31 & 32 & 36 & 37 & 39 & 40 & 45 & 46 & 49 & 51\end{array}$

$\begin{array}{lllllllllllllllllll}9 & 10 & 12 & 13 & 23 & 24 & 25 & 29 & 30 & 32 & 33 & 37 & 38 & 40 & 41 & 46 & 47 & 51 & 53\end{array}$

$\begin{array}{lllllllllllllllllll}0 & 10 & 11 & 13 & 14 & 24 & 25 & 30 & 31 & 33 & 34 & 38 & 39 & 40 & 41 & 42 & 47 & 54 & 56\end{array}$

$\begin{array}{llllllllllllllllll}0 & 1 & 11 & 12 & 14 & 15 & 19 & 25 & 26 & 31 & 32 & 34 & 35 & 39 & 40 & 42 & 43 & 48\end{array}$

$\begin{array}{llllllllllllllllll}1 & 2 & 12 & 13 & 15 & 16 & 26 & 27 & 32 & 33 & 35 & 36 & 40 & 41 & 43 & 44 & 48 & 56\end{array}$

$\begin{array}{llllllllllllllllllll}2 & 3 & 13 & 14 & 16 & 17 & 27 & 28 & 33 & 34 & 36 & 37 & 41 & 42 & 43 & 44 & 45 & 49 & 50 & 56\end{array}$

$\begin{array}{llllllllllllllllllll}3 & 4 & 14 & 15 & 17 & 18 & 28 & 29 & 34 & 35 & 36 & 37 & 38 & 42 & 43 & 45 & 46 & 48 & 50 & 53\end{array}$

$\begin{array}{llllllllllllllllllll}3 & 4 & 5 & 15 & 16 & 18 & 29 & 30 & 35 & 36 & 38 & 39 & 43 & 44 & 46 & 47 & 48 & 49 & 52 & 54\end{array}$

$\begin{array}{lllllllllllllllllll}0 & 4 & 5 & 6 & 16 & 17 & 20 & 30 & 31 & 36 & 37 & 39 & 40 & 44 & 45 & 47 & 50 & 52 & 54\end{array}$

$\begin{array}{llllllllllllllllllll}0 & 1 & 5 & 6 & 7 & 17 & 18 & 20 & 21 & 31 & 32 & 37 & 38 & 39 & 40 & 41 & 45 & 46 & 50 & 51\end{array}$

$\begin{array}{lllllllllllllllllll}1 & 2 & 6 & 7 & 8 & 18 & 21 & 22 & 32 & 33 & 38 & 39 & 41 & 42 & 46 & 47 & 51 & 52 & 54\end{array}$

$\begin{array}{llllllllllllllllllll}0 & 2 & 3 & 7 & 8 & 9 & 19 & 20 & 22 & 23 & 33 & 34 & 39 & 40 & 42 & 43 & 47 & 52 & 54 & 55\end{array}$

$\begin{array}{lllllllllllllllllll}0 & 1 & 3 & 4 & 8 & 9 & 10 & 20 & 21 & 23 & 24 & 34 & 35 & 40 & 41 & 43 & 44 & 51 & 55\end{array}$

$\begin{array}{llllllllllllllllllll}1 & 2 & 4 & 5 & 9 & 10 & 11 & 21 & 22 & 24 & 25 & 35 & 36 & 41 & 42 & 44 & 45 & 49 & 51 & 56\end{array}$

$\begin{array}{lllllllllllllllll}3 & 12 & 13 & 17 & 18 & 19 & 20 & 33 & 37 & 38 & 40 & 41 & 49 & 50 & 54 & 55 & 56\end{array}$

$\begin{array}{lllllllllllllllllllll}10 & 11 & 13 & 14 & 19 & 24 & 25 & 26 & 30 & 31 & 32 & 33 & 34 & 39 & 41 & 47 & 48 & 50 & 51 & 53 & 55\end{array}$

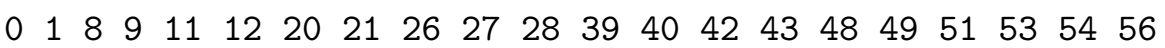

$\begin{array}{llllllllllllllllll}4 & 12 & 13 & 15 & 19 & 29 & 30 & 34 & 35 & 43 & 44 & 46 & 47 & 49 & 50 & 54 & 55 & 56\end{array}$

$\begin{array}{lllllllllllllllllll}7 & 8 & 13 & 14 & 15 & 16 & 17 & 21 & 22 & 24 & 25 & 29 & 30 & 31 & 41 & 42 & 44 & 45 & 53\end{array}$

$\begin{array}{lllllllllllllllllll}1 & 2 & 6 & 7 & 9 & 10 & 15 & 16 & 19 & 23 & 24 & 35 & 40 & 49 & 50 & 52 & 54 & 55 & 56\end{array}$

$\begin{array}{lllllllllllllllllll}4 & 5 & 7 & 8 & 19 & 24 & 25 & 27 & 28 & 36 & 41 & 42 & 44 & 45 & 48 & 50 & 51 & 53 & 55\end{array}$

$\begin{array}{lllllllllllllllll}3 & 5 & 6 & 11 & 12 & 22 & 23 & 25 & 26 & 45 & 46 & 48 & 49 & 51 & 53 & 54 & 56\end{array}$

$\begin{array}{lllllllllllllllll}0 & 2 & 8 & 16 & 19 & 21 & 22 & 32 & 36 & 38 & 39 & 47 & 48 & 50 & 51 & 53 & 55\end{array}$

Listing 2. The Color 1 Graph in a $(4,8)$-coloring of $K_{57}$. 


\section{References}

[1] G. Exoo, On Two Classical Ramsey Numbers of the Form $R(3, n)$, SIAM J. Discrete Math, 2 (1989) 488-490.

[2] G. Exoo, Announcement: On the Ramsey Numbers $R(4,6), R(5,6)$, and $R(3,12)$, Ars Combinatoria, 35 (1993) 85.

[3] G. Exoo, On the Ramsey Number $R(4,6)$, Electronic Journal of Combinatorics, Electron. J. Comb. 19 \#P66, 2012.

[4] G. Exoo, Ramsey Numbers, http://ginger.indstate.edu/ge/RAMSEY

[5] H. Fujita, A new lower bound for the Ramsey number R(4,8), arXiv 1212.1328 (2012).

[6] J. Goedgebeur, Personal communication.

[7] J. Goedgebeur and S.P. Radziszowski, New computational upper bounds for Ramsey numbers R(3,k). arXiv 1210.5826 (2012), submitted.

[8] J.G. Kalbfleisch, Chromatic Graphs and Ramseys Theorem, Ph.D. Thesis, University of Waterloo, January 1966.

[9] S. Kirkpatrick, C. D. Gelatt and M. P. Vecchi, Optimization by Simulated Annealing, Science 220 (1983) 671-680.

[10] S. P. Radziszowski, Small Ramsey Numbers, Electron. J. Combin., Dynamic Survey \#DS1, 2011. (http://www.combinatorics.org/Surveys) 